\title{
Article
}

\section{Soft Interaction between Dissolved Flexible Dendrimers: Theory and Experiment}

\author{
C. N. Likos, M. Schmidt, H. Lwen, M. Ballauff, D. Ptschke, and P. Lindner
}

Macromolecules, 2001, 34 (9), 2914-2920• DOI: 10.1021/ma001346x • Publication Date (Web): 20 March 2001

Downloaded from http://pubs.acs.org on February 12, 2009

\section{More About This Article}

Additional resources and features associated with this article are available within the HTML version:

- Supporting Information

- $\quad$ Links to the 3 articles that cite this article, as of the time of this article download

- $\quad$ Access to high resolution figures

- $\quad$ Links to articles and content related to this article

- $\quad$ Copyright permission to reproduce figures and/or text from this article

\section{View the Full Text HTML}

\section{ACS Publications}




\title{
Soft Interaction between Dissolved Flexible Dendrimers: Theory and Experiment
}

\author{
C. N. Likos,* M. Schmidt, and H. Lowen \\ Institut für Theoretische Physik II, Heinrich-HeineU niversität Düsseldorf, Universitätsstrasse 1, \\ D-40225 Düssel dorf, Germany
}

\section{Ballauff and D. Potschke}

Polymer-Institut, Universität Karlsruhe, Kaiserstrasse 12, D-76128 Karlsruhe, Germany

\section{P. Lindner}

Institut LaueLangevin, B.P. 156X, 38042 Grenoble Cedex, France

Received August 1, 2000; Revised Manuscript Received December 12, 2000

\begin{abstract}
Using small-angle neutron scattering and liquid integral equation theory, we relate the structure factor of flexible dendrimers of fourth generation to their average shape. The shape is measured as a radial density profile of monomers belonging to a single dendrimer. From that, we derive an effective interaction of Gaussian form between pairs of dendrimers and compute the structure factor using the hypernetted chain approximation. Excellent agreement with the corresponding experimental results is obtained, without the use of adjustable parameters. The present analysis thus strongly supports the previous finding that flexible dendrimers of low generation present fluctuating structures akin to star polymers.
\end{abstract}

\section{Introduction}

Dendrimers are synthetic branched macromolecules with defined structure. ${ }^{1}$ Starting from a trifunctional flexible monomer (generation 0 ), subsequent shells of trifunctional units are attached in a well-defined manner. Figure 1 displays a dendrimer of fourth generation with attached end groups at the ends of the units constituting the last generation. Thus, a treelike structure is generated, which can be viewed as an interesting intermediate between colloids and polymers: dendrimers of low generations exhibit enough degrees of conformational freedom and will thus present fluctuating structures in solution. Hence, these structures will greatly resemble star polymers or lightly branched polymers. If, on the other hand, the number of generations is increased, steric interactions between the groups located at the periphery of the molecule must result. In this case, significant back-fol ding of these groups must occur, and these structures will exhibit a more homogeneous segmental density. Hence, dendrimers of higher generation will resemble dense colloidal structures.

The average radial density distribution $T(r)$ of dendrimers is still a matter of debate. Here, the question arises as to whether dissolved dendrimers exhi bit their maximum segment density in the center or at the periphery of the molecule. The first theoretical analysis of the radial density distribution by Hervet and de Gennes came to the conclusion that dendrimers have a dense shell and hence a minimum of $T(r)$ at the center of the molecule. ${ }^{2}$ Subsequent theoretical studies, however, showed that dendrimers exhibit their maximum segment density at the center of the molecule. ${ }^{3-8}$ Only if electrostatic repulsion operates between the segments of the dendrimer is a dense shell structure to be expected. ${ }^{7}$

Small-angle scattering methods, such as small-angle X-ray scattering (SAXS) ${ }^{9}$ and small-angle neutron scat- tering (SANS), ${ }^{10}$ are suitable to investigate the radial structure of dissolved dendrimers. Up to now, however, SANS and SAXS studies of dendrimers in solution did not yet come to a final conclusion regarding the average radial density distribution $T(r)$. A survey of literature may be found in a recent review. ${ }^{11} \mathrm{~A}$ recent study of a dendrimer of seventh generation concluded that the internal structure of the molecule is rather uniform, with the end groups being preferably located at the periphery of the molecule. ${ }^{14}$ This result is in contradiction to the theoretical studies discussed above. ${ }^{3-8}$ SANS studies of dendrimers of lower generation, however, showed that the molecules under consideration have approximately a Gaussian density distribution. ${ }^{15,16}$ The chemical structure of the dendrimers investigated in refs 14-16 differ with regard to the number of generations and the nature of the end groups. The results can therefore only be compared with caution. In this respect, we also mention the simulation results of ref 8 where it was found that the conformation of dendritic molecules drastically depends on the generation number, with a soft, Gaussian-like profile resulting for small generations and a "collapsed core" with soft tails for larger generations. Neverthel ess, it must be concluded that the question of the overall structure of dissolved dendrimers has not yet found a generally accepted answer (see the discussion of this point in ref 11.)

Small-angle scattering conducted at different particle concentrations may be useful for further elucidation of this problem. Neglecting incoherent contributions, the measured scattering intensity I(q) (q: magnitude of scattering vector $\mathbf{q} ; \mathbf{q}=(4 \pi / \lambda) \sin (\theta / 2) ; \lambda$ : wavelength of radiation; $\theta$ : scattering angle) may be rendered as $s^{9,10}$

$$
\mathrm{I}(\mathrm{q})=\rho_{\mathrm{d}}\left(\bar{\rho}-\rho_{\mathrm{m}}\right)^{2} \mathrm{I}_{\mathrm{S}}(\mathrm{q}) \mathrm{S}(\mathrm{q})
$$

In eq $1, \rho_{d}=N_{d} N$ is the number density of $N_{d}$ dissolved 


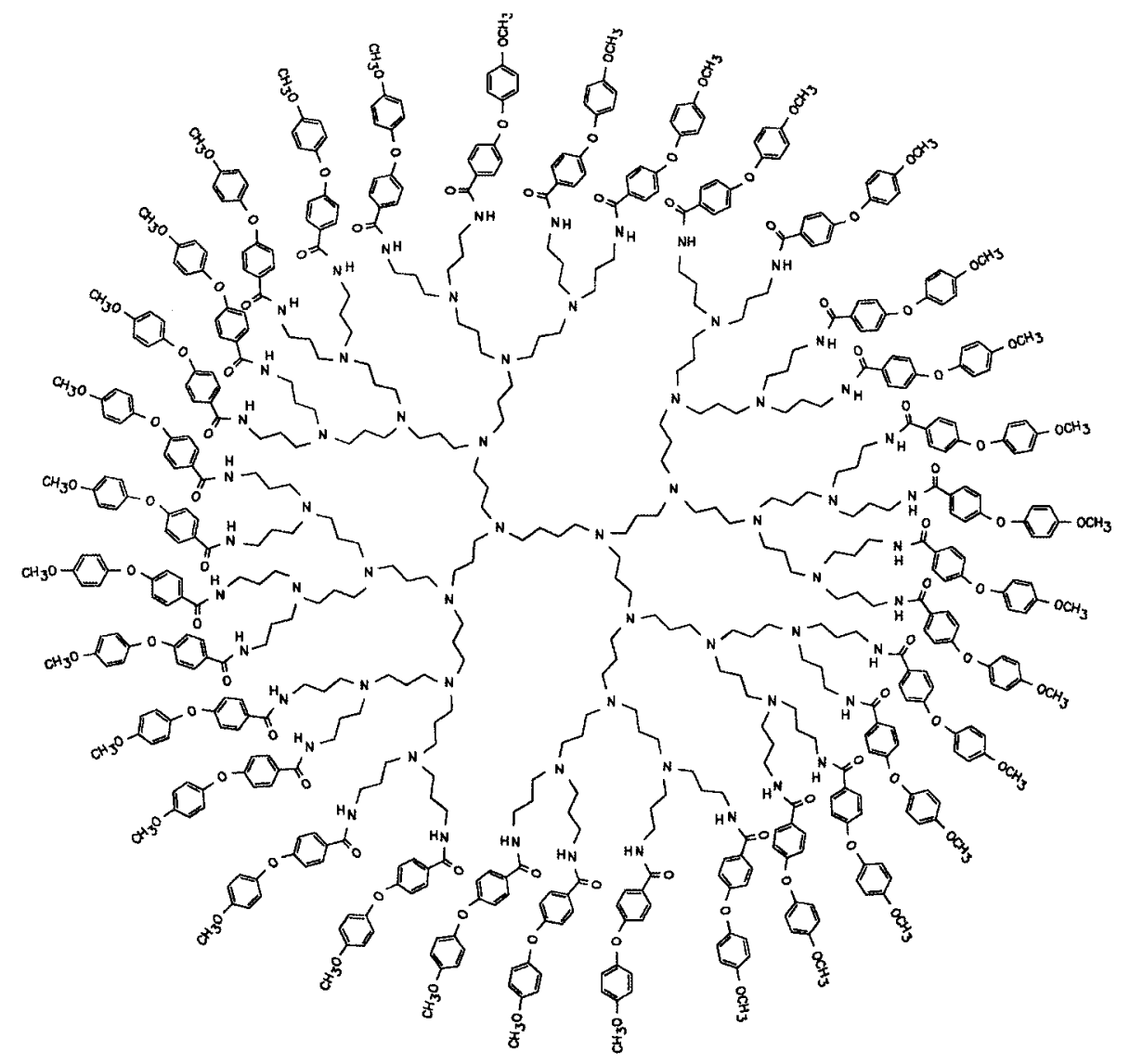

Figure 1. Structure of dendrimer G4.

dendrimers in the sample volume $\mathrm{V}$. The difference $\bar{\rho}-$ $\rho_{\mathrm{m}}$ is the contrast of the scatterer toward the solvent, whereby $\bar{\rho}$ is the average scattering length density of the scatterer and $\rho_{\mathrm{m}}$ that of the solvent. $I_{\mathrm{s}}(q)$ is the form factor of the object, a quantity directly related to the mass distribution of scattering centers within the macromolecular aggregate, as will explained below. Finally, the structure factor S(q) accounts for all interparticle correlations arising from the interactions between those. It must be emphasized that eq 1 is valid only when there are no overlaps between the particles, so that a sharp distinction between inter- and intraparticle scattering contributions can be made. Hence, it is strictly valid below the overlap concentration C*, but it can also be used above $c_{*}$ if a central core of the considered scatterers is labeled. All results we present in this work are for dilute solutions, where eq 1 is valid. Moreover, bel ow the overlap concentration no deformations of the dendrimers are expected, so that a single shape function $\mathrm{T}(\mathrm{r})$ (or form factor $\mathrm{I}_{\mathrm{s}}(\mathrm{q})$ ) can be used for all concentrations.

The effective interaction between dendrimer centers is formally defined as follows: ${ }^{12,13}$ the centers of mass of the dendrimers are kept fixed at prescribed positions, and a canonical trace is carried out over all monomer degrees of freedom. This procedure yields a constrained free energy, depending on the instantaneous configuration of the dendrimers' centers of mass. Then, the effective interaction energy between the dendrimers is $-k_{B} T$ times the logarithm of this partition function, where $k_{B}$ is Boltzmann's constant and T is the absolute temperature. When averaging over the dendrimers' positions by employing the so-defined effective Hamiltonian, the thermodynamics of the system remains invariant. In general, this procedure generates two-, three, and higher-order interactions between the centers of mass of the dendrimers. However, we will foll ow usual practice and limit our considerations to two-body potentials only; that is, we will make the usual pairpotential approximation, introducing an effective pair interaction $V_{\text {eff }}(R)$ between the centers of mass of two dendrimers separated by the distance $\mathrm{R}$. We comment on the accuracy of the pair potential approximation in section III. Since we are dealing with particles which have an overall spherical shape, this effective interaction is spherically symmetric and depends only on the magnitude of the vector connecting the two centers.

The structure factor $S(q)$ is directly related to the pair correlation function $g(R)$ and hence to the total interaction potential of the solute molecules. ${ }^{17}$ Once $V_{\text {eff }}(R)$ is known, the calculation of $g(R)$ and $S(q)$ follows from the solution of any of the rich variety of so-called liquid integral equation theories. ${ }^{17} \mathrm{~F}$ or infinite dilution $\mathrm{S}(\mathrm{q})$ $=1$. Hence, from an experimental point of view, eq 1 shows that $I_{s}(q)$ may be obtained from SANS or SAXS data which have been suitably extrapolated to vanishing concentration. ${ }^{10}$

The structure factor $\mathrm{S}(\mathrm{q})$ gives direct information on the steepness of the repulsion of the dissolved particles: in the case of hard spheres, a pronounced structuring of the solution will occur, which leads to a marked maximum of $\mathrm{S}(\mathrm{q}) .{ }^{17}$ If the dissolved particles interact via soft repulsion, the maximum of $S(q)$ is decreased. Recently, the problem of S(q) of star polymers has been addressed. ${ }^{18,19}$ Here, it could be shown that star polymers may be looked upon as ultrasoft colloidal particles. The predictions of theory have met with gratifying success when compared to SANS data mea- 
sured for concentrated solutions of star polymers. 20,21

Up to now, only a few experimental studies have addressed the problem of the structure factor of dissolved dendrimers. Ramzi et al. studied concentrated solutions of dendrimers by SANS. ${ }^{22}$ In the case of uncharged dendrimers, these workers found a marked depression of the peak of $\mathrm{S}(\mathrm{q})$ despite the strong decrease of the structure factor at low q. They concluded that the absence of a peak of $S(q)$ is related to the softness of interaction, which results from the high internal flexibility of the dendritic structures. If charges are added to the segments of the dissolved dendrimers, a strong peak of $S(q)$ is generated which is clearly traced back to the screened Coulombic interactions of the now charged species (cf. also ref 23.) A SANS study by Topp et al. showed that the dendrimers under consideration exhibited only a weak maximum of $S(q)$, in particular at lower generation. ${ }^{24}$ At high concentrations the evaluation of data according to eq 1 did not lead to meaningful results, which indicates a considerable change of the conformation of the dendrimers in this regime. In the dilute regime, no change of the intrinsic structure of the dendrimers has been observed. This regime has been studied in refs 15 and 16 . The data of S(q) have been interpreted in terms of a simplified model, treating the dendrimers as effective hard spheres. It is hence evident that the problem of $\mathrm{S}(\mathrm{q})$ of dissolved dendrimers is not yet understood quantitatively.

In this work, we wish to apply a simple theory which allows us to calculate the effective interaction between dendrimer centers using the measured density profile of an isolated dendrimer as a starting point. It turns out that the interaction has a Gaussian form. Subsequently, we employ it to calculate theoretically the structure factor $\mathrm{S}(\mathrm{q})$ of dissolved dendrimers at various concentrations using the hypernetted chain (HNC) approximation. The consequences of the internal flexibility on the measured structure factor $\mathrm{S}(\mathrm{q})$ will be discussed explicitly. Moreover, a quantitative comparison with the experimental data presented in ref 16 will be given, showing excellent agreement. It will be demonstrated that the radial density distribution $T(r)$ derived from scattering experiments may directly be used to calculate S(q).

\section{Theory}

The starting point for the theory is the monomer density profile $\rho(r)$ around the center of mass of an isolated dendrimer. The experiments of refs 15 and 16 offer a direct access to this quantity, through the socalled shape function $T(r)$. The scattering intensity $I_{S}(q)$ from a solution of dendrimers at infinite dilution has been written as (see eq 18 in ref 15 )

$$
\mathrm{I}_{\mathrm{S}}(\mathrm{q})=\mathrm{T}^{2}(\mathrm{q})
$$

meaning that $\mathrm{T}^{2}(\mathrm{q})$ is the form factor of the dendrimer and hence $T(q)$ the F ourier transform of $T(r)$. Hence, as al ready pointed out in ref $15, T(r)$ is a "shape function" that describes the way in which $\rho(r)$ varies in space. This shape function is dimensionless; hence, we may write

$$
\rho(r)=\rho_{0} T(r)
$$

where $\rho_{0}$ is a constant having dimensions of density (length ${ }^{-3}$ ) and which will be specified now. Equation 3 together with eq 2 of ref 15 implies

$$
\int \mathrm{d} \mathbf{r} \rho(\mathrm{r})=\rho_{0} \mathrm{~V}_{\mathrm{p}}
$$

where $\mathrm{V}_{\mathrm{p}}$ is the measured partial volume of the solute molecule. On the other hand, the integral of $\rho(r)$ has to yield the total number of monomers $\mathrm{N}$ within the dendrimer, and this fact together with eq 4 implies

$$
\rho(r)=\frac{N}{V_{p}} T(r)
$$

The shape function $T(r)$ is determined by an inverse Fourier transform of $\mathrm{T}(\mathrm{q})$, the latter being given by eq 20 of ref 15. Ignoring the "tail" $\left(\mathrm{aq}^{2}+\mathrm{bq}\right) \exp \left(-\mathrm{dq}^{2}\right)$ there, which only has a minor effect on the profile in the neighborhood $r \cong 0$, we have

$$
T^{2}(q)=V_{p}^{2} \exp \left(-q^{2} R_{g}^{2} / 3\right)
$$

From eq 6 and after an inverse F ourier transformation, we readily obtain

$$
T(r)=V_{p}\left(\frac{3}{2 \pi R_{g}^{2}}\right)^{3 / 2} \exp \left(-\frac{3 r^{2}}{2 R_{g}^{2}}\right)
$$

It can be easily seen that $\int \mathrm{dr} T(\mathrm{r})=\mathrm{V}_{\mathrm{p}}$, in agreement with eq 2 of ref 15

From eqs 5 and 7 we obtain the monomer density as

$$
\rho(r)=N\left(\frac{3}{2 \pi R_{g}^{2}}\right)^{3 / 2} \exp \left(-\frac{3 r^{2}}{2 R_{g}^{2}}\right)
$$

We now assume that two such dendrimers are kept with their centers of mass at a separation $\mathbf{R}$ apart and wish to calculate the ensuing interaction potential. Let us assume that the monomer-monomer interaction potential is given by some function $v\left(\boldsymbol{r}_{1}-\mathbf{r}_{2}\right)$ where $\boldsymbol{r}_{1}$ and $\mathbf{r}_{2}$ are the positions of the two monomers. In the mean-fied d approximation, i.e., ignoring the correlations and possible deformations of the dendrimers, the total interaction potential $\mathrm{V}_{\text {eff }}(\mathbf{R})$ can be approximated by a double integral over the unperturbed density profiles times the monomer-monomer interaction kernel. ${ }^{25}$ This approximation should hold when the dendrimers are not too deeply interpenetrating and reads as

$\mathrm{V}_{\text {eff }}(\mathbf{R})=\iint \mathrm{d} \mathbf{r}_{1} \mathrm{~d} \mathbf{r}_{2} \rho\left(\mathbf{r}_{1}\right) \rho\left(\mathbf{r}_{2}-\mathbf{R}\right) \mathrm{v}\left(\mathbf{r}_{1}-\mathbf{r}_{2}\right)$

We now make the simplest possible assumption and model the monomer-monomer interaction by a delta function (contact repulsion):

$$
\mathrm{v}\left(\mathbf{r}_{1}-\mathbf{r}_{2}\right)=\mathrm{v}_{0} \mathrm{k}_{\mathrm{B}} \mathrm{T} \delta\left(\boldsymbol{r}_{1}-\mathbf{r}_{2}\right)
$$

introducing the excluded-volume parameter $v_{0}$. Equations 9 and 10 then yield

$$
\mathrm{V}_{\text {eff }}(\mathbf{R})=\mathrm{V}_{0} \mathrm{k}_{\mathrm{B}} \mathrm{T} \int \mathrm{d} \mathbf{r} \rho(\mathbf{r}) \rho(\mathbf{r}-\mathbf{R})
$$

In this approximation, the interaction is proportional to the convolution of the monomer density of a single dendrimer with itself. But each density profile has the form $\mathrm{N}(\alpha / \pi)^{3 / 2} \exp \left(-\alpha r^{2}\right)$, i.e., $\mathrm{N}$ times a normalized Gaussian with width parameter $\alpha$. It is known that the convolution of a normalized Gaussian having width $\alpha$ 
with itself is again a normalized Gaussian, but with width $\alpha / 2$. Hence, eqs 8 and 11 yield the final result:

$$
V_{\text {eff }}(R)=N^{2}\left(\frac{3}{4 \pi R_{g}^{2}}\right)^{3 / 2} V_{0} k_{B} T \exp \left(-\frac{3 R^{2}}{4 R_{g}^{2}}\right)
$$

The effective potential depends only on the magnitude $R=|\mathbf{R}|$ of the center-to-center separation, because of rotational symmetry. Note that the interaction potential of eq 12 is identical to the Flory-Krigbaum effective interaction potential between the centers of mass of two polymer chains. ${ }^{26}$ The fact that we are dealing with dendrimers enters into the relation between $N$ and $R_{g}$. For long, self-avoiding chains, $\mathrm{R}_{\mathrm{g}} \propto \mathrm{N}^{3 / 5}$ holds; in the present study this is not the case, and we determine both quantities from experiment.

The important feature is that the resulting interaction has a Gaussian form:

$$
\mathrm{V}_{\mathrm{eff}}(\mathrm{R})=\epsilon \exp \left(-\frac{\mathrm{R}^{2}}{\sigma^{2}}\right)
$$

with

$$
\epsilon=N^{2}\left(\frac{3}{4 \pi R_{g}^{2}}\right)^{3 / 2} v_{0} k_{B} T
$$

and

$$
\sigma=\sqrt{\frac{4}{3}} \mathrm{R}_{\mathrm{g}}
$$

The task is now to calculate $\epsilon$ from the experimental data. The number of monomers per dendrimer is $\mathrm{N}=$ 94 (see Figure 1) where the aromatic end groups are also included in the counting as "single monomers".

Next we need an estimate for the excluded-volume parameter $v_{0}$. For this, we set $v_{0}=I_{0}{ }^{3}$, where $I_{0}$ is the monomer length. Taking the realistic value $I_{0}=0.4 \mathrm{~nm}$ for the latter quantity, we obtain

$$
\mathrm{v}_{0}=0.064 \mathrm{~nm}^{3}
$$

The above values together with the experimentally determined gyration radius $R_{g}=1.85 \mathrm{~nm}$ yield

$$
\epsilon=10.42 \mathrm{k}_{\mathrm{B}} \mathrm{T}
$$

The corresponding value for linear chains is about $2 \mathrm{k}_{\mathrm{B}} \mathrm{T}$. ${ }^{28}$ Hence, the energy barrier for the dendrimers at hand is about 5 times higher as that for linear chains, a result that is physically reasonable as dendrimers are more compact objects than chains. A general study of the structural and thermodynamic properties of a system of particles interacting by a Gaussian potential (the "Gaussian core model", GCM) has been presented recently. ${ }^{27}$ The GCM shows no freezing for $\epsilon \lesssim 100$ (see ref 27); hence, we conclude that the system of dendrimers at hand will remain fluid at all concentrations.

Next, we express the density of dendrimers in units that are more convenient. Since there are $\mathrm{N}_{\mathrm{d}}$ dendrimers in the volume $\mathrm{V}$, the density $\rho_{\mathrm{d}}$ of dendrimers is $\mathrm{N}_{\mathrm{d}} N$. In ref 15 , the volume fraction $\phi$ was used which is

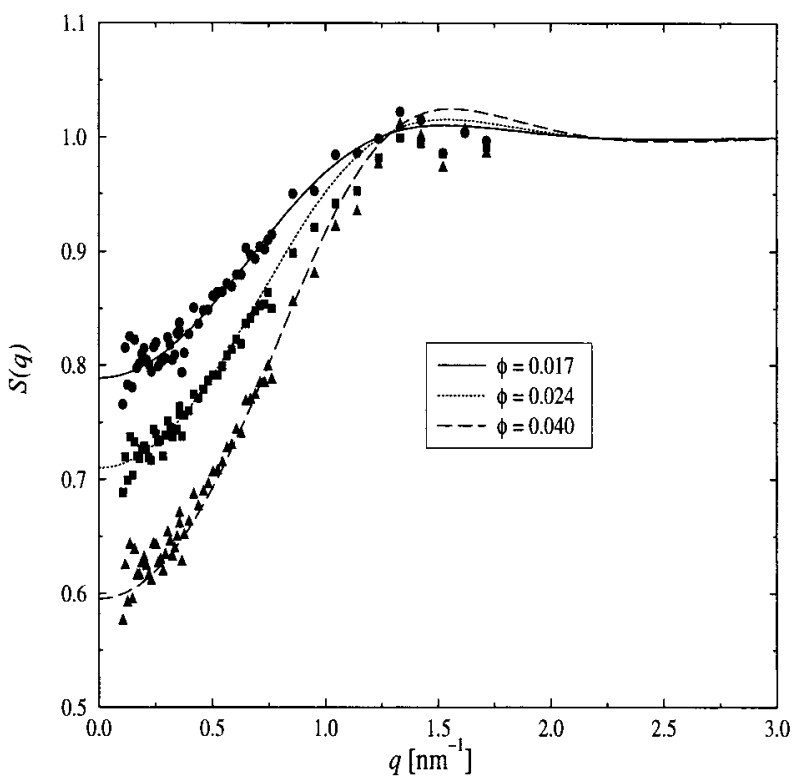

Figure 2. Experimentally measured (points) and theoretically calculated (curves) structure factors $S(q)$ for solutions of dendrimers at three different concentrations. The theoretical curves have been obtained by employing a Gaussian effective interaction between the dendrimers.

related to $\rho_{\mathrm{d}}$ by

$$
\phi=\rho_{\mathrm{d}} \mathrm{V}_{\mathrm{p}}
$$

To suppress the dependence on $v_{p}$, it is natural to use as the unit of length the parameter $\sigma$ of the pair potential and to introduce a dimensionless measure of the density, namely

$$
\eta=\frac{\pi}{6} \rho_{\mathrm{d}} \sigma^{3}
$$

which may be regarded as an effective volume fraction of the dissolved dendrimers. From eqs 15, 18, and 19 we obtain

$$
\eta=\frac{\pi}{6}\left(\frac{4}{3}\right)^{3 / 2 \mathrm{R}_{\mathrm{g}}{ }^{3}} \frac{\mathrm{V}_{\mathrm{p}}}{} \phi=0.338 \phi
$$

where the known values $R_{g}=1.85 \mathrm{~nm}$ and $V_{p}=15.1$ $\mathrm{nm}^{3}$ have been used. ${ }^{15}$

For every value of $\eta$, the hypernetted chain (HNC) equation ${ }^{17}$ for a system interacting via $\beta v(r)=10.42 \times$ $\exp \left(-r^{2} / \sigma^{2}\right)$ was solved, and the structure factor $S(q)$ was obtained as a function of $q \sigma$. Using eq 15 , we obtain $\sigma$ $=2.136 \mathrm{~nm}$. This is in close agreement with the effective radius of $2.4 \mathrm{~nm}$ estimated in ref 15 by a simple model valid only for dilute solutions. In what is to follow the $\mathrm{q}$-scale was reexpressed in $\mathrm{nm}^{-1}$ units.

\section{Results and Discussion}

As can be seen from Figure 2, the Gaussian interaction potential yields excellent agreement between theory and experiment at all three concentrations measured. Moreover, this is achieved without the use of free fit parameters, and it is physically reasonable that soft, interpenetrable objects such as dendrimers interact by means of a correspondingly soft interaction. The boundedness of the effective interaction at zero separations between the dendrimer centers is also physically cor- 


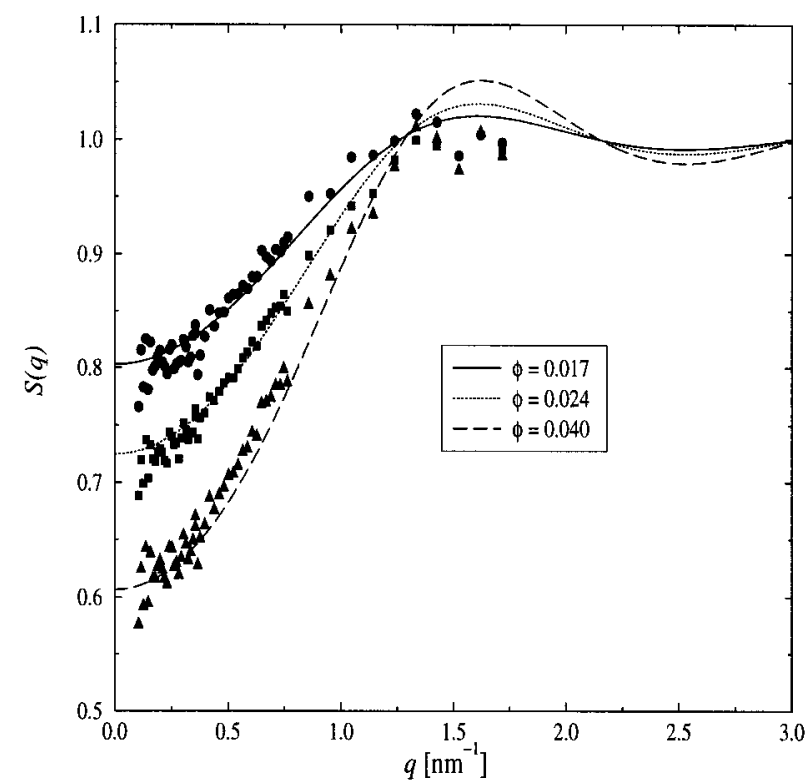

Figure 3. Same as Figure 2, but now the theoretical curves have been obtained by employing a hypothetical hard-sphere (HS) interaction between the dendrimers, with a HS diameter $\sigma_{\mathrm{HS}}=1.95 \mathrm{R}_{\mathrm{g}}$.

rect: configurations where the centers of mass of two dendrimers coincide are possible, without violation of excluded-volume conditions; hence, the effective potential does not diverge at the origin. This is quite analogous to the case of polymer chains, where the effective interaction is known to be Gaussian. ${ }^{13,28}$

The precise numerical value $\epsilon=10.42 \mathrm{k}_{\mathrm{B}} \mathrm{T}$ of the energy barrier, eq 17, yields an optimal agreement with the experimental data. However, small deviations from this value can al so be tolerated, and by comparing with the SANS data, we have established the limits in which this parameter can vary as $8.0 \lesssim \epsilon \lesssim 12.0$, yielding a corresponding tol erance interval $0.370 \mathrm{~nm} \lesssim \mathrm{I}_{0} \lesssim 0.425$ $\mathrm{nm}$ for the "effective monomer length" $I_{0}$.

A prominent characteristic of the Gaussian potential at the reduced temperatures considered here (and also at higher ones) is its property to yiel d structure factors which do not show any pronounced peak with increasing density, in direct contradistinction with hard, diverging interactions such as the hard-sphere (HS) potential. ${ }^{27}$ To demonstrate this point, we have also attempted to fit the experimental data using a hypothetical HS interaction between the dendrimers, with the HS diameter $\sigma_{\mathrm{HS}}$ as a fit parameter. The results are shown in Figure 3. The best fit at the lowest concentration is obtained by the choice $\sigma_{\mathrm{HS}}=1.95 \mathrm{R}_{\mathrm{g}}$, which is thereafter kept constant. The reason for keeping $\sigma_{\mathrm{HS}}$ unchanged in the dilute regime is that the shape and size of the dendrimers themselves remain unchanged. This also makes the comparison between the GCM and the HS models fair, as the parameters of both potentials are held density-indepenedent. Besides, a putative better fit with a density-dependent effective hard-sphere diameter $\sigma_{\mathrm{HS}}$ would only be another way of saying that the true effective interaction is not a hard-sphere one. It can be seen that the fit quality worsens with increasing density, as the HS interaction yields a too high peak as well as a structure factor at low q values which lies bel ow the experimental data. However, as we are in the very dilute regime, the strong differences between the structure factors produced by these two interactions

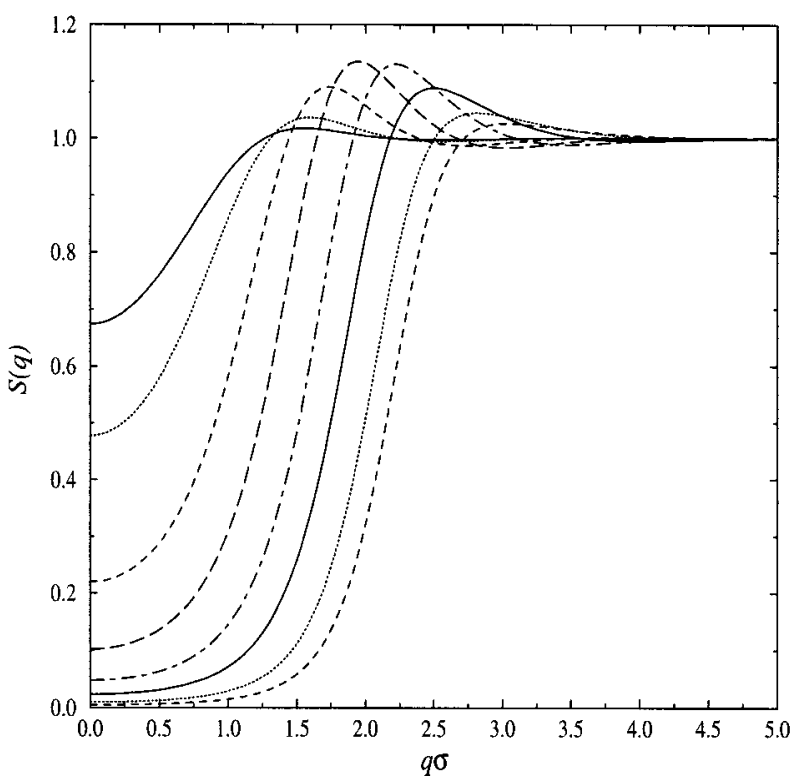

Figure 4. Structure factor of the Gaussian core model at energy $\epsilon=10 \mathrm{k}_{\mathrm{B}} \mathrm{T}$ as a function of the "packing fraction" $\eta$ given in eq 19. From left to right: $\eta=0.01,0.02,0.05,0.10,0.20$, $0.40,1.00$, and 2.00 .

(Gaussian and HS) are not very pronounced. These differences become evident only if one looks at higher densities. The result found here is therefore in agree ment with the findings of ref 15 . There it has been found that modeling the structure factor in terms of a simple hard-sphere ansatz leads to a satisfactory description of the data in the region of lowest volume fractions.

It is therefore evident that a more stringent test of theory can only be achieved by SANS studies conducted at much higher concentrations. Experiments using the G4 and G5 dendrimer used in refs 15 and 16 are under way. Here we present further evidence for the validity of the proposed interaction by comparing with and by offering a theoretical explanation for data already existing in the literature. ${ }^{22,24}$

In Figure 5 of ref 22, Ramzi et al. display the experimentally determined structure factor for a very wide concentration range of dendrimers, ranging from dilute to above the overlap concentration. It is seen that $\mathrm{S}(\mathrm{q})$ is deprived of any significant structure; it has a weak peak of height $\sim 1.1$ at intermediate concentrations, and thereafter the peak disappears and S(q) becomes a monotonic function of $q$. At the same time, this phenomenon is accompanied by a monotonic reduction of the osmotic compressibility of the solution, the latter being proportional to the $\mathrm{q} \rightarrow 0$ limit of $\mathrm{S}(\mathrm{q})$. This is precisely the behavior of $\mathrm{S}(\mathrm{q})$ of the Gaussian core model.$^{27}$ The latter has a freezing and reentrant melting transition with increasing density at energies $\epsilon \geq$ $100 \mathrm{~K}_{\mathrm{B}} \mathrm{T}$, meaning that the liquid $\mathrm{S}(\mathrm{q})$ has an anomal ous dependence on the density: the height of its principal maximum grows up to a certain density, and then it diminishes again. As the temperature is increased, the anomaly in S(q) remains, but the height of the principal peak becomes smaller. In order to provide a semiquantitative comparison with the data of ref 22 , we show in Figure 4 the evolution of $S(q)$ of the GCM with density for an energy barrier $\epsilon=10 \mathrm{~K}_{\mathrm{B}} \mathrm{T}$ and packing fractions $\eta=0.01-2.00$, corresponding to $\phi \lesssim 0.67$. A striking similarity with the results of ref 22 can be easily seen.

Similar conclusions hold when we compare our pre dictions with the scattering data of Topp et al., Figures 
7 and 8 in ref 24. There, the S(q)'s from samples of two different dendrimers are displayed; the same anomal ous dependence of $\mathrm{S}(\mathrm{q})$ on the concentration is seen, with the additional feature that the S(q)'s from the dendrimers with the larger generation number have higher peaks than their counterparts from the smaller dendrimers at the same concentration. In our language, a larger generation number, which implies a larger monomer number $\mathrm{N}$, corresponds to a higher energy barrier $\epsilon$; see eq 14 . This automatically causes $S(q)$ to develop stronger peaks for larger generation numbers.

Of particular interest is the shape of $\mathrm{S}(\mathrm{q})$ for very high concentrations which, as can be seen in refs 22 and 24 , is a monotonic function of $\mathrm{q}$. This behavior can be fully understood in the framework of the Gaussian interaction potential. It has been recently shown that bounded potentials in general show at high densities a particular "mean-fiel d behavior".27,29,30 The direct correlation function $c(r)$ in this limit becomes equal to $-\beta v(r)$, where $v(r)$ is the interaction potential at hand and $\beta=\left(\mathrm{k}_{\mathrm{B}} \mathrm{T}\right)^{-1}$. F or the case of the Gaussian potential, this implies that the structure factor at the high density limit takes the form 27,30

$$
\mathrm{S}(\mathrm{q})=\frac{1}{1+\pi^{3 / 2} \beta \epsilon \rho \sigma^{3} \exp \left[-(\mathrm{Q} \sigma / 2)^{2}\right]}
$$

or, more generally, for any bounded potential $v(r)^{29,30}$

$$
\mathrm{S}(\mathrm{q})=\frac{1}{1+\beta \rho \tilde{\mathrm{V}}(\mathrm{q})}
$$

where $\tilde{v}(q)$ is the Fourier transform of $v(r)$. Equation 21 shows that, for the GCM, S(q) has the form of a "smoothed out" step function. It is small at low q's and approaches unity at high q's, as $\tilde{v}(q) \rightarrow 0$ there. The same conclusion holds, actually, for any bounded potential whose Fourier transform is a monotonically decaying, nonnegative function of $\mathrm{q}^{29}$ as can be seen from eq 22.

The anomalous behavior of the peak heights of the structure factor would be impossible if the assumed interaction between dendrimers were diverging at the origin as any power law. Indeed, power-law systems are known to undergo a freezing transition, this implying that the corresponding $\mathrm{S}(\mathrm{q})$ develops stronger and stronger peaks with increasing density until, ultimately, freezing occurs when the principal peak reaches the quasi-universal Hansen-Verlet value $2.85 .{ }^{31}$ Hence, effective dendrimer-dendrimer interactions with such kinds of divergence at the origin can be immediately ruled out. This argument, however, does not rule out all diverging interactions. Indeed, the peak-height anomaly has also been observed in the framework of a theoretical treatment of star polymers, ${ }^{19}$ whose effective interaction is diverging logarithmically at the origin. This behavior, which has also been experimentally seen, 32 is again intimately related to the reentrant melting behavior of these systems. ${ }^{33,34}$ In fact, it has al ready been suggested ${ }^{24}$ that dendrimers may resemble star polymers bel ow the critical arm number $f_{c}=34$ at which crystallization is marginally possible . $^{33-35} \mathrm{How}-$ ever, at variance with the Gaussian interaction, the star-star effective potential 20 does not have a meanfield high-density limit, and the structure factor of stars never becomes a monotonic function of $q$, even at extremely high densities, ${ }^{19}$ a feature which appears for bounded interactions only. In this respect, there is a difference between the effective interaction between the central monomers of two dendrites or two stars (which diverges) and that between their centers of mass (which does not).

On purely theoretical grounds, the underlying assumptions leading to the derivation of the logarithmic interaction between star polymers do not hold for dendrimers. Unlike stars, dendrimers do not obey a power-law dependence of the density profile around the center; they show no self-similarity, captured in the Daoud-Cotton blob model of the stars, 36 and they are at least 1 order of magnitude smaller than star polymers, with the implication that many of the notions of polymer physics based on long chains and universality cannot be automatically carried over to dendrimers.

We conclude this section with a remark on the accuracy of the pair potential approximation. Though many-body forces between the dendrimers are necessarily present in the solution, there are good reasons to believe that their effect can be neglected to a very good approximation. This belief is based on one hand on the corresponding findings on star polymers. ${ }^{37}$ By a direct measurement of the triplet forces, it was established there that these have a very small effect on the total force on a star center, and this only shows up at concentrations considerably beyond the overlap value. On the other hand, a recent simulational work by Louis et al. ${ }^{28}$ reached a similar conclusion for single chains. There, it was found that the pair potential yielding the correct thermodynamics of the system remains densityindependent up to the overlap concentration and displays only a very weak density dependence above the latter. This density dependence is, of course, just another way to formulate the many-body effects. Once more, they were found to be of minor significance. Barring any dramatic al terations in the conformations of single dendrimers upon increasing density (such as collapsing), we expect the Gaussian pair potential picture to capture the salient characteristics of the behavior of this system at all concentrations.

\section{Summary and Conclusions}

By using the density profile of an isolated dendrimer and the experimentally determined characteristics of the macromolecules as input, we derived an effective interaction between the dendrimers' centers of mass which is Gaussian in form. By direct comparison with experimental data at dilute solutions, we show that this interaction provides an excellent description of the measured structure factor. Moreover, it reproduces correctly the as of now unexplained trends and features of experimental structure factors at higher densities. A direct comparison with SANS data at high concentrations is, evidently, of crucial importance for further putting the proposed theory into a strong test. Work along these lines is currently in progress.

Acknowledgment. The authors gratefully acknowledge financial support by the Deutsche Forschungsgemeinschaft and by the Bundesministerium für Forschung und Technologie.

\section{References and Notes}

(1) Fischer, M.; Vögtle, F. Angew. Chem., Int. Ed. Engl. 1999, $38,884$.

(2) de Gennes, P. G.; Hervet, H. J . Phys. (Paris) 1993, 44, L 351.

(3) Lescanec, R. L.; Muthukumar, M. Macromolecules 1990, 23, 2280. 
(4) Mansfield, M. L.; Klushin, L. L. Macromolecules 1993, 26, 4262.

(5) Murat, M.; Grest, G. S. Macromolecules 1996, 29, 1278.

(6) Boris, D.; Rubinstein, M. Macromolecules 1996, 29, 7251.

(7) Welch, P.; Muthukumar, M. Macromol ecules 1998, 31, 5892.

(8) Lue, L. Macromolecules 2000, 22, 2266.

(9) Feigin, L. A.; Svergun, D. I. Structure Analysis by SmallAngle X-Ray Scattering and Neutron Scattering; Plenum Press: New York, 1987.

(10) Higgins, J . S.; Benoît, H. C. Polymers and Neutron Scattering; Clarendon Press: Oxford, 1994.

(11) Ballauff, M. In Structure and Dynamics of Polymer and Colloidal Systems; NATO ASI Series; Pecora, R., Borsali, R., Eds.; Kluwer Academic Publishers: Dordrecht, , in press.

(12) Löwen, H.; Madden, P. A.; Hansen, J .-P.J . Chem.' Phys. 1993 $98,3275$.

(13) Likos, C. N. Phys. Rep., in press.

(14) Topp, A.; Bauer, B. J .; Klimash, J . W.; Spindler, R.; Tomalia, D. A.; Amis, E. J. Macromolecules 1999, 32, 7226 and references therein.

(15) Pötschke, D.; Ballauff, M.; Lindner, P.; Fischer, M.; Vögtle, F. Macromol. Chem. Phys. 2000, 201, 330.

(16) Pötschke, D.; Ballauff, M.; Lindner, P.; Fischer, M.; Vögtle, F. Macromolecules 1999, 32, 4079.

(17) Hansen, J.-P.; MacDonald, I. R. Theory of Simple Liquids, 2nd ed.; Academic: London, 1986.

(18) J usufi, A.; Watzlawek, M.; Löwen, H. Macromolecules 1999, $32,4470$.

(19) Watzlawek, M.; Löwen, H.; Likos, C. N. J . Phys.: Condens. Matter 1998, 10, 8189.

(20) Likos, C. N.; Löwen, H.; Watzlawek, M.; Abbas, B.; J ucknischke, O.; Allgaier, J .; Richter, D. Phys. Rev. Lett. 1998, 80, 4450.

(21) Stellbrink, J.; Allgaier, J .; Monkenbusch, M.; Richter, D.; Lang, A.; Likos, C. N.; Watzlawek, M.; Löwen, H.; Ehlers, G.; Schleger, P. Prog. Colloid Polym. Sci. 2000, 115, 88.
(22) Ramzi, A.; Scherrenberg, R.; Brackman, J .; J oosten, J .; Mortensen, K. Macromolecules 1998 31, 1621.

(23) Micali, N.; Monsú Scolaro, L.; Romeo, A.; Lombardo, D.; Lesieur, P.; Mallamace, F. Phys. Rev. E 1998, 58, 6229.

(24) Topp, A.; Bauer, B. J .; Prosa, T. J .; Scherrenberg, R.; Amis, E. J. Macromolecules 1999, 32, 8923.

(25) Graf, H.; Löwen, H. Phys. Rev. E 1998, 57, 5744.

(26) Flory, P. J .; Krigbaum, W. R. J . Chem. Phys. 1950, 18, 1086.

(27) Lang, A.; Likos, C. N.; Watzlawek, M.; Löwen, H. J . Phys.: Condens. Matter 2000, 12, 5087.

(28) Louis, A. A.; Bolhuis, P. G.; Hansen, J .-P. Phys. Rev. Lett. 2000, 85, 2522 and references therein.

(29) Likos, C. N.; Lang, A.; Watzlawek, M.; Löwen, H. Phys. Rev. E 2001, 63, 031206.

(30) Louis, A. A.; Bolhuis, P. G.; Hansen, J.-P. Phys. Rev. E 2000, 62, 7961.

(31) Hansen, J .-P.; Verlet, L. Phys. Rev. 1969, 184, 151.

(32) J ucknischke, O. Untersuchung der Struktur von Sternpolymeren in Lösung mit Neutronenkleinwinkelstreuung. Doctoral Thesis, Westfälische Wilhelms-Universität Münster, 1995 (in German).

(33) Watzlawek, M.; Likos, C. N.; Löwen, H. Phys. Rev. Lett. 1999, 82,5289

(34) Watzlawek, M. Phase Behavior of Star Polymers; Shaker Verlag: Aachen, 2000

(35) Witten, T. A.; Pincus, P. A.; Cates, M. E. Europhys. Lett. 1986 2, 137.

(36) Daoud, M.; Cotton, J. P. J . Phys. (Paris) 1982, 43, 531.

(37) von Ferber, C.; J usufi, A.; Likos, C. N.; Löwen, H.; Watzlawek, M. Eur. Phys. J . E 2000, 2, 311.

MA001346X 